RECORDS OF PHARMACEUTICAL
AND BIOMEDICAL SCIENCES

\title{
Antibiotics Profile and $\beta$-Lactamases in Pseudomonas aeruginosa
}

\author{
Ali A. Abdelrahman ${ }^{a}$, Alaa El-Din M.S. Hosny ${ }^{b}$, Dalia M. Hamed $^{c}$, Samira Zakeer ${ }^{a^{*}}$ \\ ${ }^{a}$ Department of Microbiology and Immunology, Faculty of Pharmacy, Suez Canal University, Ismailia, \\ Egypt; ${ }^{b}$ Department of Microbiology and Immunology, Faculty of Pharmacy, Cairo University, Cairo, Egypt; \\ ${ }^{c}$ Department of Quality Control, VACSERA, Giza, Egypt.
}

Received on: 01-3-2021

Revised on: 16-3-2021

Accepted on: 20-3-2021

Correspondence Author:

Tel: +201092574619

E-mail address:

samirazakeer2007@ hotmail.com

\begin{abstract}
Pseudomonas aeruginosa most strains are motile by means of a single polar flagellum. It usually lives in moist environments and uses a wide range of organic compounds for growth, giving it an exceptional ability to penetrate plant and animal tissues. P.aeruginosa colonizes human body sites, mainly the moist areas, such as the ear, nasal mucosa and throat, as well as stools. It contains 163 known or predicted porin proteins based on its genome sequence, 64 are found as part of 3 families of porins, the OprD-specific porin family, the TonB-dependent gated porin family and the OprM efflux family. $\beta$-lactamases are normally located in the periplasm, but it has been detected during the antipseudomonal treatment in the sputum, these enzymes are highly released from high level producers in the lungs. MBL are characterized with zinc ion as a cofactor and with a separate heritage, the common feature of MBLs is the principal zinc binding HistidineHistidine-XAspartic acid in the active site, which coordinates the arrangement of two $\mathrm{H} 2 \mathrm{O}$ molecules that are important in the hydrolysis. The chelation of zinc by EDTA or mercaptopropionic acid, impairs $\beta$-lactam hydrolysis and restores susceptibility to Carbapenems. MBLs have a wide active site, which let all $\beta$-lactams to accomodate in there, except Aztreonam. $\beta$-lactamase inhibitors such as clavulanic acid, Tazobactam and Sulbactam are also hydrolysed by MBLs. This review was done to figure out the antimicrobial profile and the different $\beta$-lactamases present in Pseudomonas aeruginosa.
\end{abstract}

Keywords: Pseudomonas aeruginosa, metallo $\beta$-lactamases, Carbapenems

\section{Introduction}

Pseudomonas aeruginosa is a non-fermentative, opportunistic, aerobic Gram-negative rod, measuring 0.5 to $0.8 \mu \mathrm{m}$ by 1.5 to $3.0 \mu \mathrm{m}$. P.aeruginosa strains produce two types of soluble pigments, pyoverdin and pyocyanin. Pyocyanin (from "pyocyaneus") refers to "blue pus", which is characteristic for suppurative infections caused by
P.aeruginosa (Murray \& American Society for, 1999). P.aeruginosa has a large genome containing $6.26 \mathrm{Mbp}$ (encoding 5567 genes) compared to 4.64 Mbp (4279 genes) in Escherichia coli (Lambert, 2002). It causes nosocomial respiratory tract infections including ventilator-associated pneumonia (VAP), dermatitis, soft tissue infections, bacteraemia, bone and joint infections, gastrointestinal infections and a variety 
of systemic infections, particularly in immunosuppressed patients (AIDS) or patients with severe burns or cancer (Lambert, 2002). P.aeruginosa has many virulence factors, including flagella, pili, lipopolysaccharides, alginate, alkaline protease, elastase, phospholipase $\mathrm{C}$ and exotoxin (Murray \& American Society for, 1999). The AmpC gene of the inducible $\beta$-lactamase is present in all P.aeruginosa strains. The enzyme is present and normally located in the periplasm, but it has been detected during the antipseudomonal treatment in the sputum (Davies \& Bilton, 2009). This enzyme is highly released from high-level producers in the lungs and would protect low-level producers by reducing the local concentration of certain $\beta$-lactam antibiotics, like Cephalothin or Ampicillin. The most popular $\beta$-lactamases are PSE-1 and PSE-4. TEM and OXA enzymes are not effective against Carbapenems,

Oxyimino-Aminothiazole Cephalosporins (Ceftazidime, Cefepime, Cefpirome and Monobactams. PER1 $\beta$-lactamases, TEM, SHV and OXA ESBLs, arbapanamases and the metallo- $\beta$ lactamases are $\beta$-lactamases that give wider resistance (Deplano et al., 2005; Farrell et al., 2007). PER-1, an Ambler's molecular class A $\beta$-lactamase was first discovered in 1991 in France then recognized in Turkey and distributed in Poland, Japan and Romania (Quinteira \& Peixe, 2006) and reveals high resistance level to Ceftazidime, but it has little in vitro effect on Piperacillin. OXA-type ESBLs presence is quite frequent among Pseudomonas (van Belkum et al., 2007). There are five groups of these enzymes, namely OXA group I (including OXA-5; OXA-7; OXA-10 and its derivatives: OXA-11, OXA-14, OXA-16, OXA-17, OXA-74; OXA-13 and its derivatives: OXA-19, OXA-28), OXA group II (including OXA-2; OXA3; OXA-15; OXA-20), OXA group III (including OXA-1, OXA-4, OXA-30, ESBLs, OXA-31), OXA group IV (defined by OXA-9) and OXA group V (containing LCR-1). In addition, OXA-18 does not belong to any of these groups (van Belkum et al., 2007).

\section{Antibiotic resistance to Pseudomonas aeruginosa:}

P.aeruginosa characterized by high resistances to a wide range of antimicrobials (Gales, Jones, Turnidge, Rennie, \& Ramphal, 2001). Its intrinsic resistance to many antimicrobial agents and the ability to develop multidrug resistance and mutational acquired resistance to antibiotics through chromosomal mutations results in a serious therapeutic problems (Gales et al., 2001) The $\beta$ lactams inhibit the peptidoglycan assembling transpeptidases located on the outer face of the cytoplasmic membrane, some $\beta$-lactams are active against $P$.aeruginosa, most Cephalosporins are not active against $P$.aeruginos $a$ but, Ceftazidime, a third generation agent and Cefepime, a fourth-generation agent, have excellent and the same activity. Carbapenems is a class of broad-spectrum $\beta$-lactam antibiotics, Meropenem has slightly greater activity against P.aeruginosa (Ayalew, Nambiar, Yasinskaya, \& Jantausch, 2003; Baldwin, LysengWilliamson, \& Keam, 2008; Shah \& Narang, 2005). Ciprofloxacin is the most active fluoroquinolone against P.aeruginosa and the Aminoglycosides inhibit protein synthesis by binding to the $30 \mathrm{~S}$ subunit of the ribosome and they have been used in the treatment of these infections (Hauser \& Sriram, 2005). Aminoglycosides are active components of antipseudomonal chemotherapy used in the treatment of many infections, mainly pulmonary infections in cystic fibrosis patients with aerosolized tobramycin. The first aminoglycoside-resistant isolates reported are from the 1960s, and nowadays they are widely distributed all over the world, mainly in Europe and Latin America (Wolter et al., 2008). Finally, the Polymyxins bind to phospholipids in the cytoplasmic membrane, destroying its barrier function. However, P.aeruginosa adaptive ability causes difficulties for the sensitivity of microbial identification methods and it has become necessary to develop genotypebased characterization systems capable of accurately identifying these bacteria. The molecular identification eliminates the problem of different phenotypes and allows for more accurate identification of bacteria (Drancourt et al., 2000).

\section{Restricted uptake of antibiotics:}

All of the antibiotics to reach their targets have to cross the cell wall, the inability of antibiotics to accumulate in the organism is due to the efflux pumps that results in restricted permeability of the outer membrane and the efficient removal of antibiotic molecules. P.aeruginosa outer membrane is an important barrier of antibiotics which has low permeability (Dinesh, Grundmann, Pitt, \& Römling, 2003), excluding the larger molecules. $\beta$-lactams and quinolones can only cross the outer membrane through the channels provided by porin proteins because they are small hydrophilic 


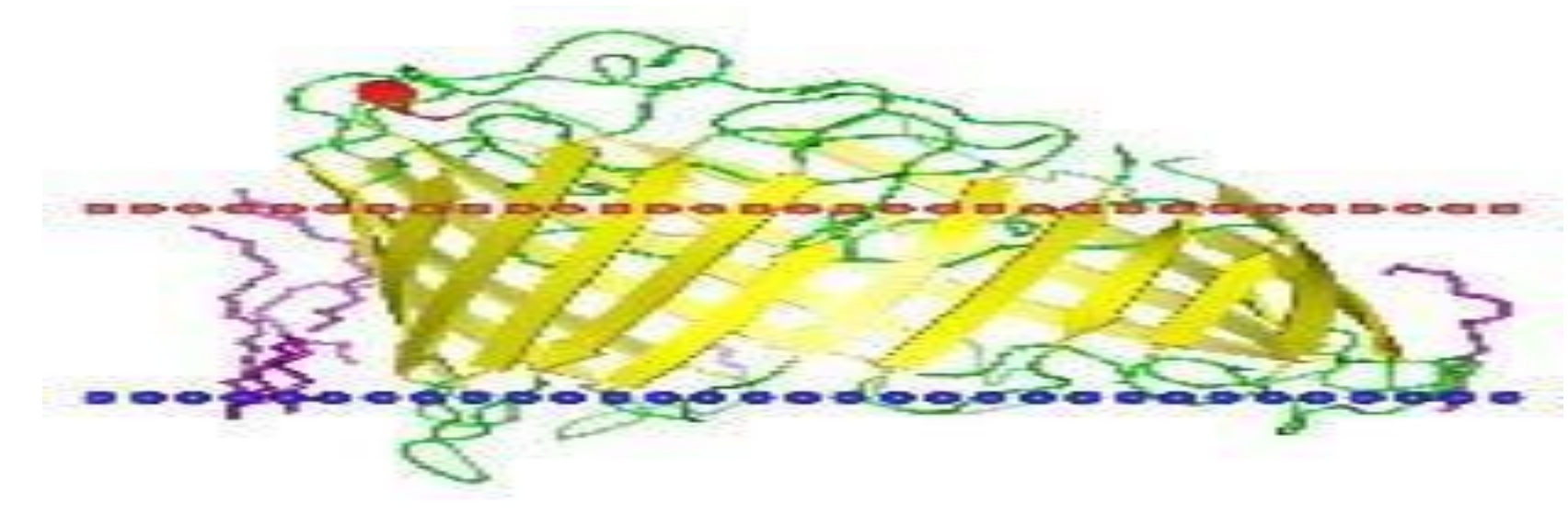

\section{Transmembrane \\ antiparallel $\beta$-strands}

Figure 1. The structure of the outer membrane porin OprD. (Source of picture is: opm.phar.umich.edu/protein.php?pdbid=2odi)

molecules. Porins are characterized by having a transmembrane anti-parallel $\beta$-strands that wrap into a barrel, and this $\beta$-barrel is embedded into the outer membrane bilayer (Figure 1.).

\section{4. $\beta$-lactamases:}

$\beta$-lactamases are enzymes which have the ability to catalyse the hydrolysis of an amide bond in the $\beta$ lactam ring of antibiotics belonging to the penicillin/cephalosporin family that can create an ineffective antimicrobial (Abraham \& Chain, 1988). They are of a very important theory in the clinical approach because of their clinical importance and their ecological and evolutionary interest, they are also thought to share a common ancestor with the DD-peptidases, which form peptide cross-links with the peptidoglycan network during cell wall synthesis (Kelly et al., 1986). Practically both the $\beta$-lactamases and the DD-peptidases are very similar in the acylation step (Pratt, 2002). However, the acyl intermediate of the $\beta$-lactamases undergoes deacylation much more rapidly and is therefore very efficient at destroying the antibiotic and protecting the peptidoglycan synthesis (Sun, Nukaga, Mayama, Braswell, \& Knox, 2003) The inactivation of a $\beta$ lactam antibiotic by a $\beta$-lactamase and were classified into classes A, B, C and D $\beta$-lactamases (Caselli et al., 2001).

\subsection{Class A $\beta$-lactamases}

Class A enzymes contain the $\beta$-lactamases SHV, TEM, CARB/PSE, OHIO, ROB-1 and PC1. According to the Bush classification scheme (Bush \& Jacoby, 2010), depending mainly on biochemical substrates properties and reactions with inhibitors, this resulted in hydrolysis of Penicillins, Cephalosporins or Carbenicillins. Class A enzymes mechanism of action includes the nucleophilic attack by Ser70 after activation by Lys 73 or Glu166 on the carbonyl carbon of the $\beta$-lactam ring resulting in the acyl enzyme intermediate (Majiduddin, Materon, \& Palzkill, 2002). Class A $\beta$-lactamases include four motifs, S130-X-N, S70-X-X-K, K234$\mathrm{T} / \mathrm{S}-\mathrm{G}$ and the $\mathrm{W}$-loop, they are found in the majority of the active-site pocket of the S70-X-X-K motif includes the active site serine at position 70 and a lysine at position 73 (Ambler, 1980). In the back donation of the carbonyl proton, this occurs via a hydrogen bond network involving a water molecule, Lys73 and Lys234, a component of the KT/S-G motif. Lys234 stabilises Ser130 (a component of the S-X-N motif) through hydrogen bonding (Matagne, Lamotte-Brasseur, \& Frère, 1998). Deacylation and regeneration of the $\beta$ lactamase may happen via a water molecule activation by Glu166. This activated water molecule had attacked the carbonyl carbon of the acyl enzyme intermediate, and back donation of a 


\section{Abdelrahman et al.}

proton to the Ser70 resulted in a regenerated enzyme (Majiduddin et al., 2002)The Glu166 in the W-loop region of Class A b-lactamases is important for catalysis and is responsible for the activation of the water molecule that promotes a good deacylation of the acyl-enzyme intermediate.

\subsection{Class B $\beta$-lactamases}

Metallo- $\beta$-lactamases are classified as group 3 enzymes because they have the ability to hydrolyse Penicillins, Cephalosporins and Carbapenems, but are resistant mainly to all usual $\beta$-lactam inhibitors (Bush \& Jacoby, 2010). This category does not hydrolyse Aztreonam (Ambler, 1980) classified metallo- $\beta$-lactamases as class B enzymes. Class B $\beta$ lactamases require divalent cations, primarily zinc for activity and are inhibited by metal chelators and the production of these enzymes is mainly constitutive (Majiduddin et al., 2002).

The genes for class B $\beta$-lactamases are either chromosomal or plasmid-borne and are found on integrons (Laraki et al., 1999). They have identified Class B metallo- $\beta$-lactamases in both Gram-positive and Gram negative bacteria. They are found in many clinically important species such as Acinetobacter spp. and Pseudomonas aeruginosa. The most popular ClassB $\beta$-lactamases belonged to the IMP and VIM families (Majiduddin et al., 2002).

These enzymes are classified into three functional subgroups, B1, B2 and B3, depending on metal requirements. In subclass $\mathrm{B} 1$, it includes most metallo- $\beta$-lactamases, it was revealed that $\mathrm{Zn} 1$ is coordinated very tightly and $\mathrm{Zn} 2$ is coordinated very loosely (Fabiane et al., 1998). The B2 functional subgroup contains two zinc sites each with high binding affinity similarity (Crowder et al., 1996). VIM and IMP classes are the only MBLs that have been found to have three subgroups, which are as follows: VIM-7 group, VIM-2 group, VIM-1 group and IMP-12, IMP-1, IMP-2 group, IMP-1 group, while GIM and SPM have been found in a restricted geographical area (Johnson, Arduino, Stine, Johnson, \& Harris, 2007).

\subsection{Class $C \beta$-lactamases}

These enzymes were known as cephalosporinases, they can be discriminated depending mainly on their primary structure and active site serine (Ambler, 1980). Bush et al. 1995 had described class C enzymes as group $1 \beta$-lactamases. They are

\section{Rec. Pharm. Biomed. Sci. 5 (2), 74-79, 2021}

only produced by Gram-negative bacteria the Enterobacteriaceae, but have also been produced by Pseudomonas and Aeromonas. The genetic encoding of these enzymes are highly usually chromosomally encoded (Sanschagrin, Couture, \& Levesque, 1995).

The main representative of this group is AmpC (Majiduddin et al., 2002). The phenotype study revealed that they are distinguished by the resistance to a variety of Cefoxitin, Cefotetan, Ceftazidime, Penicillins, $\beta$-lactamase inhibitors and Carbapenems (Giuliani, Docquier, Riccio, Pagani, \& Rossolini, 2005).

\subsection{Class D B-lactamases}

Class A, C and D $\beta$-lactamases were found to share structural similarities with the target of $\beta$-lactam antibiotics, the DD-peptidases, and they emerged from the same ancestral enzyme (Koch, 2003). It was revealed that the DNA sequence similarity between the class D $\beta$-lactamase and class $A$ and $C$ is applied to three main regions around the active site (Majiduddin et al., 2002). Class D $\beta$-lactamases differ significantly from Class $\mathrm{A}$ and $\mathrm{C}$ enzymes by the usage of a carboxylated lysine side chain for catalysis (Golemi, Maveyraud, Vakulenko, Samama, \& Mobashery, 2001).

The Class D deacylation step of catalysis is greatly slower than the acylation step because the oxacillinase enzymes activate a water molecule for hydrolysis less actively (Sun et al., 2003). The first class $\mathrm{D} \beta$-lactamases were belonging to as oxacillinases because they commonly hydrolyse the isoxazolyl penicillins, oxacillin and cloxacillin, two to four times quicker than classical Penicillins such as Penicillin G (Sun et al., 2003).

\section{Conclusion:}

Metallo- $\beta$-lactamases are considered as enzymes because they can hydrolyse Penicillins, Cephalosporins and Carbapenems and they are present in many important clinical species of Pseudomonas aeruginosa.

\section{Conflict of interest:}

The authors report no declaration of conflict of interest. 


\section{References:}

Abraham, E., \& Chain, E. (1988). An enzyme from bacteria able to destroy penicillin. 1940. Reviews of infectious diseases, 10(4), 677-678.

Ambler, R. P. (1980). The structure of betalactamases. Philos Trans $R$ Soc Lond B Biol Sci, 289(1036), 321-331. doi:10.1098/rstb.1980.0049

Ayalew, K., Nambiar, S., Yasinskaya, Y., \& Jantausch, B. A. (2003). Carbapenems in pediatrics. Therapeutic drug monitoring, 25(5), 593-599.

Baldwin, C. M., Lyseng-Williamson, K. A., \& Keam, S. J. (2008). Meropenem. Drugs, 68(6), 803838.

Bush, K., \& Jacoby, G. A. (2010). Updated functional classification of beta-lactamases. Antimicrob Agents Chemother, 54(3), 969-976. doi:10.1128/aac.01009-09

Caselli, E., Powers, R. A., Blasczcak, L. C., Wu, C. Y., Prati, F., \& Shoichet, B. K. (2001). Energetic, structural, and antimicrobial analyses of beta-lactam side chain recognition by beta-lactamases. Chem Biol, $\quad 8(1), \quad 17-31 . \quad$ doi:10.1016/s10745521(00)00052-1

Davies, J. C., \& Bilton, D. (2009). Bugs, biofilms, and resistance in cystic fibrosis. Respiratory care, 54(5), 628-640.

Deplano, A., Denis, O., Poirel, L., Hocquet, D., Nonhoff, C., Byl, B., . . Struelens, M. J. (2005). Molecular characterization of an epidemic clone of panantibiotic-resistant Pseudomonas aeruginosa. $J$ Clin Microbiol, 43(3), 1198-1204. doi:10.1128/jcm.43.3.1198-1204.2005

Dinesh, S., Grundmann, H., Pitt, T., \& Römling, U. (2003). European-wide distribution of Pseudomonas aeruginosa clone C. Clinical microbiology and infection, 9(12), 1228-1233.

Drancourt, M., Bollet, C., Carlioz, A., Martelin, R., Gayral, J.-P., \& Raoult, D. (2000). 16S ribosomal DNA sequence analysis of a large collection of environmental and clinical unidentifiable bacterial isolates. Journal of clinical microbiology, 38(10), 3623-3630.

Fabiane, S. M., Sohi, M. K., Wan, T., Payne, D. J.,
Bateson, J. H., Mitchell, T., \& Sutton, B. J. (1998). Crystal structure of the zinc-dependent betalactamase from Bacillus cereus at 1.9 A resolution: binuclear active site with features of a mononuclear enzyme. Biochemistry, 37(36), 12404-12411. doi:10.1021/bi980506i

Farrell, P., Joffe, S., Foley, L., Canny, G. J., Mayne, P., \& Rosenberg, M. (2007). Diagnosis of cystic fibrosis in the Republic of Ireland: epidemiology and costs. Ir Med J, 100(8), 557-560.

Gales, A., Jones, R., Turnidge, J., Rennie, R., \& Ramphal, R. (2001). Characterization of Pseudomonas aeruginosa isolates: occurrence rates, antimicrobial susceptibility patterns, and molecular typing in the global SENTRY Antimicrobial Surveillance Program, 1997-1999. Clinical Infectious Diseases, 32(Supplement_2), S146-S155.

Giuliani, F., Docquier, J. D., Riccio, M. L., Pagani, L., \& Rossolini, G. M. (2005). OXA-46, a new class $\mathrm{D}$ beta-lactamase of narrow substrate specificity encoded by a blaVIM-1-containing integron from a Pseudomonas aeruginosa clinical isolate. Antimicrob Agents Chemother, 49(5), 1973-1980. doi:10.1128/aac.49.5.1973-1980.2005

Golemi, D., Maveyraud, L., Vakulenko, S., Samama, J. P., \& Mobashery, S. (2001). Critical involvement of a carbamylated lysine in catalytic function of class D beta-lactamases. Proc Natl Acad $\begin{array}{llllll}\text { Sci } U & S & A & \text { 98(25), } & \text { 14280-14285. }\end{array}$ doi:10.1073/pnas.241442898

Hauser, A. R., \& Sriram, P. (2005). Severe Pseudomonas aeruginosa infections: tackling the conundrum of drug resistance. Postgraduate medicine, 117(1), 41-48.

Johnson, J. K., Arduino, S. M., Stine, O. C., Johnson, J. A., \& Harris, A. D. (2007). Multilocus sequence typing compared to pulsed-field gel electrophoresis for molecular typing of Pseudomonas aeruginosa. J Clin Microbiol, 45(11), 3707-3712. doi: $10.1128 / \mathrm{jcm} .00560-07$

Kelly, J. A., Dideberg, O., Charlier, P., Wery, J.-P., Libert, M., Moews, P., . . Joris, B. (1986). On the origin of bacterial resistance to penicillin: comparison of a beta-lactamase and a penicillin target. Science, 231(4744), 1429-1431. 


\section{Abdelrahman et al.}

Koch, A. L. (2003). Bacterial wall as target for attack: past, present, and future research. Clin Microbiol Rev, 16(4), 673-687. doi:10.1128/cmr.16.4.673-687.2003

Lambert, P. (2002). Mechanisms of antibiotic resistance in Pseudomonas aeruginosa. Journal of the royal society of medicine, 95(Suppl 41), 22.

Laraki, N., Galleni, M., Thamm, I., Riccio, M. L., Amicosante, G., Frère, J. M., \& Rossolini, G. M. (1999). Structure of In31, a blaIMP-containing Pseudomonas aeruginosa integron phyletically related to In5, which carries an unusual array of gene cassettes. Antimicrob Agents Chemother, 43(4), 890901. doi:10.1128/aac.43.4.890

Majiduddin, F. K., Materon, I. C., \& Palzkill, T. G. (2002). Molecular analysis of beta-lactamase structure and function. Int J Med Microbiol, 292(2), 127-137. doi:10.1078/1438-4221-00198

Matagne, A., Lamotte-Brasseur, J., \& Frère, J. M. (1998). Catalytic properties of class A betalactamases: efficiency and diversity. Biochem J, 330 ( Pt 2)(Pt 2), 581-598. doi:10.1042/bj3300581

Murray, P. R., \& American Society for, M. (1999). Manual of clinical microbiology. Washington: ASM Press.

Pratt, R. (2002). Functional evolution of the serine $\beta$ lactamase active site. Journal of the Chemical Society, Perkin Transactions 2(5), 851-861.

Quinteira, S., \& Peixe, L. (2006). Multiniche screening reveals the clinically relevant metallo-
Rec. Pharm. Biomed. Sci. 5 (2), 74-79, 2021

beta-lactamase VIM-2 in Pseudomonas aeruginosa far from the hospital setting: an ongoing dispersion process? Appl Environ Microbiol, 72(5), 3743-3745. doi:10.1128/aem.72.5.3743-3745.2006

Sanschagrin, F., Couture, F., \& Levesque, R. C. (1995). Primary structure of OXA-3 and phylogeny of oxacillin-hydrolyzing class D beta-lactamases. Antimicrob Agents Chemother, 39(4), 887-893. doi:10.1128/aac.39.4.887

Shah, D., \& Narang, M. (2005). Meropenem. Indian pediatrics, 42(5), 443-450.

Sun, T., Nukaga, M., Mayama, K., Braswell, E. H., \& Knox, J. R. (2003). Comparison of betalactamases of classes $\mathrm{A}$ and $\mathrm{D}$ : 1.5-A crystallographic structure of the class D OXA-1 oxacillinase. Protein Sci, 12(1), 82-91. doi: 10.1110/ps.0224303

van Belkum, A., Tassios, P. T., Dijkshoorn, L., Haeggman, S., Cookson, B., Fry, N. K., . . . Struelens, M. (2007). Guidelines for the validation and application of typing methods for use in bacterial epidemiology. Clin Microbiol Infect, 13 Suppl 3, 1-46. doi:10.1111/j.14690691.2007.01786.x

Wolter, D. J., Acquazzino, D., Goering, R. V., Sammut, P., Khalaf, N., \& Hanson, N. D. (2008). Emergence of carbapenem resistance in Pseudomonas aeruginosa isolates from a patient with cystic fibrosis in the absence of carbapenem therapy. Clin Infect Dis, 46(12), e137-141. doi:10.1086/588484 